\title{
Macroinvertebrados de hojarasca y suelo en selva baja caducifolia y zonas perturbadas
}

\author{
Comparative study macroinvertebrate in litter and soil in deciduous \\ forest and disturbed areas by communication routes
}

\author{
Oscar A. Barreto-García1, Rubén D. Guevara-Gutiérrez ${ }^{1 *}$, José L. Olguín-López"1, \\ O. Raúl Mancilla-Villa², Edna Karen Medina Valdovinos², Juan Eduardo Murillo Hernández
}

\begin{abstract}
RESUMEN
Con el objetivo de conocer la composición de macroinvertebrados en hojarasca y suelo, en el presente estudio se analizó el impacto del cambio de uso de suelo por efecto de la construcción de vías de comunicación (ZP) sobre comunidades de macroinvertebrados y su presencia en Selva Baja Caducifolia (SCB) en la cuenca hidrográfica Casa de Piedra, municipio de Autlán de Navarro, Jalisco-México. Los muestreos se realizaron de forma aleatoria en hojarasca y suelo a una profundidad de 0-30 cm, las unidades de muestreo fueron cuadrantes de 30 × $30 \mathrm{~cm}$. Se contabilizó e identificó el número de individuos colectados, describiendo, analizando y determinando a nivel taxonómico de orden y familia, usando claves dicotómicas, colecciones de referencia y guías entomológicas ilustradas. En las comunidades identificadas se estimó la riqueza específica y diversidad biológica a través de los índices de Margalef y Shannon. La unidad de muestreo (UM) con mayor riqueza específica y diversidad biológica de macroinvertebrados fue la SBC en hojarasca con $\mathrm{Da}=3,59$ y H' $=2,98$ respectivamente. Del inventario obtenido se identificó solo los grupos funcionales: ingenieros del suelo, detritívoros, herbívoros, depredadores.
\end{abstract}

Palabras clave: Macroinvertebrados, suelo, hojarasca, selva baja caducifolia, zonas perturbadas, grupos funcionales.

\begin{abstract}
To study the impact of changing land use due to the construction of roads on macroinvertebrate communities and their presence in lowland analyzed caducifolia in the watershed House, municipality of Autlan, Jalisco-Mexico, the composition of macroinvertebrates in litter and soil was determined. Samples were taken randomly in litter and soil to a depth of $30 \mathrm{~cm}$; sampling units were quadrants of $30 \times 30 \mathrm{~cm}$. The individuals collected were counted, described and identified to the family level using dichotomous keys, reference collections and illustrated identification guides. The species richness and diversity of the communities identified were estimated using the Margalef and Shannon indices. The sampling unit (SU) with greatest species richness and biological diversity of macroinvertebrates was the $S B C$ in litter with $D a=3.59$ and $H^{\prime}=2.98$. Respectively inventory obtained functional groups was identified: soil engineers, detritus, herbivores, predators.
\end{abstract}

Keywords: Macroinvertebrates, soil, leaves, deciduous forest, disturbed areas, functional groups.

\section{Introducción}

El estudio de los macroinvertebrados edáficos considera la división de aquellos que se encuentran en hojarasca y el suelo; en ambos casos, los macroinvertebrados incluyen a los invertebrados visibles a simple vista mayores de $2 \mathrm{~mm}$, que pueden incluir más de un millar de especies en un solo ecosistema, alcanzar densidades y biomasas de más de un millón de individuos y más de una tonelada por hectárea respectivamente (Brown et al., 2001). Algunos de estos organismos son llamados ingenieros del suelo ya que transforman las propiedades y producen estructuras físicas que modifican la disponibilidad o accesibilidad de recursos para otros organismos (Jones et al., 1994),

1 Departamento de Ecología y Recursos Naturales del Centro Universitario de la Costa Sur de la Universidad de Guadalajara. Guadalajara, México.

2 Departamento de Producción Agrícola del Centro Universitario de la Costa Sur de la Universidad de Guadalajara. Guadalajara, México.

* Autor por correspondencia: oscar.29barreto@gmail.com, rguevara@cucsur.udg.mx.

Fecha de Recepción: 27 Octubre, 2016.

Fecha de Aceptación: 13 Enero, 2018. 
afectando directa e indirectamente la abundancia o estructura de otras comunidades de organismos (Jones et al., 1997).

La relevancia de estudiar la macrofauna edáfica radica en ser identificada como reguladores de muchos procesos del ecosistema, puesto que tienen efectos positivos en la conservación de la estructura del suelo; actúan sobre el microclima, la humedad y la aireación, activan o inhiben la función de microorganismos y están involucrados en la conservación y ciclado de nutrientes a través de la descomposición de la hojarasca (Lavelle et al., 1993; Salamanca y Chamorro, 1994; Wolters y Ekschmitt, 1997). Por ello, los macroinvertebrados del suelo se consideran como indicadores de la calidad del suelo, así como organismos indispensables en la provisión de los bienes y servicios proporcionados por los agroecosistemas (Lavelle et al., 2006). En la hojarasca interactúan con microorganismos mediante relaciones mutualistas externas e internas, contribuyendo al reciclaje de nutrimentos a través de la descomposición de la hojarasca; son reconocidos como organismos de alta sensibilidad a perturbaciones, lo que los convierte en buenos indicadores del impacto humano sobre el ambiente (Guinchard y Robert, 1991).

Brussard (1998) identifica en la fauna edáfica su gran diversidad, la redundancia funcional en los procesos ecosistémicos y el poco conocimiento que se tiene de ellos ante lo cual los agrupa en gremios y/o grupos funcionales en consideración de-la historia natural, forma de alimentarse, alimento principal, micro hábitat utilizado y la ecofisiología. Hooper et al. (2002), Lavorel y Garnier (2002), identificaron estas comunidades por sus propiedades bióticas, su ecosistema y por su adaptación a cambios en el ambiente provocados por disturbios naturales o de origen humano.

Basado en Zaldívar et al. (2007) referente a la compresión de ecosistemas naturales y perturbados, en el presente estudio se asocian patrones de funcionamiento de comunidades de macroinvertebrados en suelo y hojarasca para ambos ecosistemas considerando la Selva Baja Caducifolia (SBC) en comparación con zonas perturbados (ZP) por efecto de construcción de vías de comunicación. Para el primer ecosistema Rzedowski (1991) lo considera como una unidad con alta diversidad, elevado endemismo y de gran importancia en México por registrarse en esta vegetación $60 \%$ de las especies florísticas para el país.

\section{Materiales y Métodos}

\section{Área de estudio}

El estudio se desarrolló en la cuenca hidrográfica "Arroyo El Coajinque" situada en el Municipio de Autlán de Navarro en el Estado de Jalisco-México, sobre la que se está construyendo la carretera que une los municipios de Autlán de Navarro y Villa Purificación (Figura 1) en las coordenadas $104^{\circ} 23^{\prime} 41^{\prime \prime}$ a $104^{\circ} 28^{\prime} 13^{\prime \prime}$ 'Oeste y $19^{\circ} 44^{\prime} 48^{\prime \prime}$ a $19^{\circ} 46^{\prime} 18^{\prime \prime}$ Norte, sobre una superficie de 2.064 hectáreas, ubicada a una altura promedio sobre el nivel del mar de $1.102 \mathrm{~m}$; esta cuenca se distribuye sobre una superficie de 7.255,9 ha. Los tipos de suelos dominantes son: Feozem háplico, Fluvisol eútrico, Litosol, Regosol dístrico y Regosol eútrico; la vegetación y uso de suelo son: Bosque de Encino, Selva Baja Caducifolia, Bosque de Encino Abierto, Agricultura y Pasto. El clima es semiseco con otoño, invierno y primavera secos y semicálidos, sin cambio térmico invernal bien definido; la temperatura media anual es de $23.5^{\circ} \mathrm{C}$ y precipitación media anual de 719.8 milímetros.

\section{Métodos}

Los muestreos se realizaron mensualmente en Selva Baja Caducifolia (SBC) y zona perturbada por construcción de vías de comunicación (ZP) durante un año, consideradas como unidades de muestreo (UM) establecidos al azar apoyados de la tabla de números aleatorios y un mapa cuadriculado del área de estudio, el criterio de selección del sitio fue la homogeneidad y representatividad de las coberturas de cada UM, la superficie considerada por muestreo fueron cuadros de 30 por $30 \mathrm{~cm}$, considerando una profundidad de $0-30 \mathrm{~cm}$; se tomaron muestras en los estratos de hojarasca y suelo por cada UM y llevadas a laboratorio para la extracción manual de macroinvertebrados y posterior identificación; los individuos colectados fueron cuantificados y clasificados taxonómicamente en orden y familia mediante el apoyo de guías entomológicas ilustradas, claves dicotómicas y colecciones de referencia (Laboratorio de zoología, Departamento de Ecología y Recursos Naturales del Centro Universitario de la Costa Sur de la Benemérita Universidad de Guadalajara). Para conocer la homogeneidad y/o heterogeneidad de 


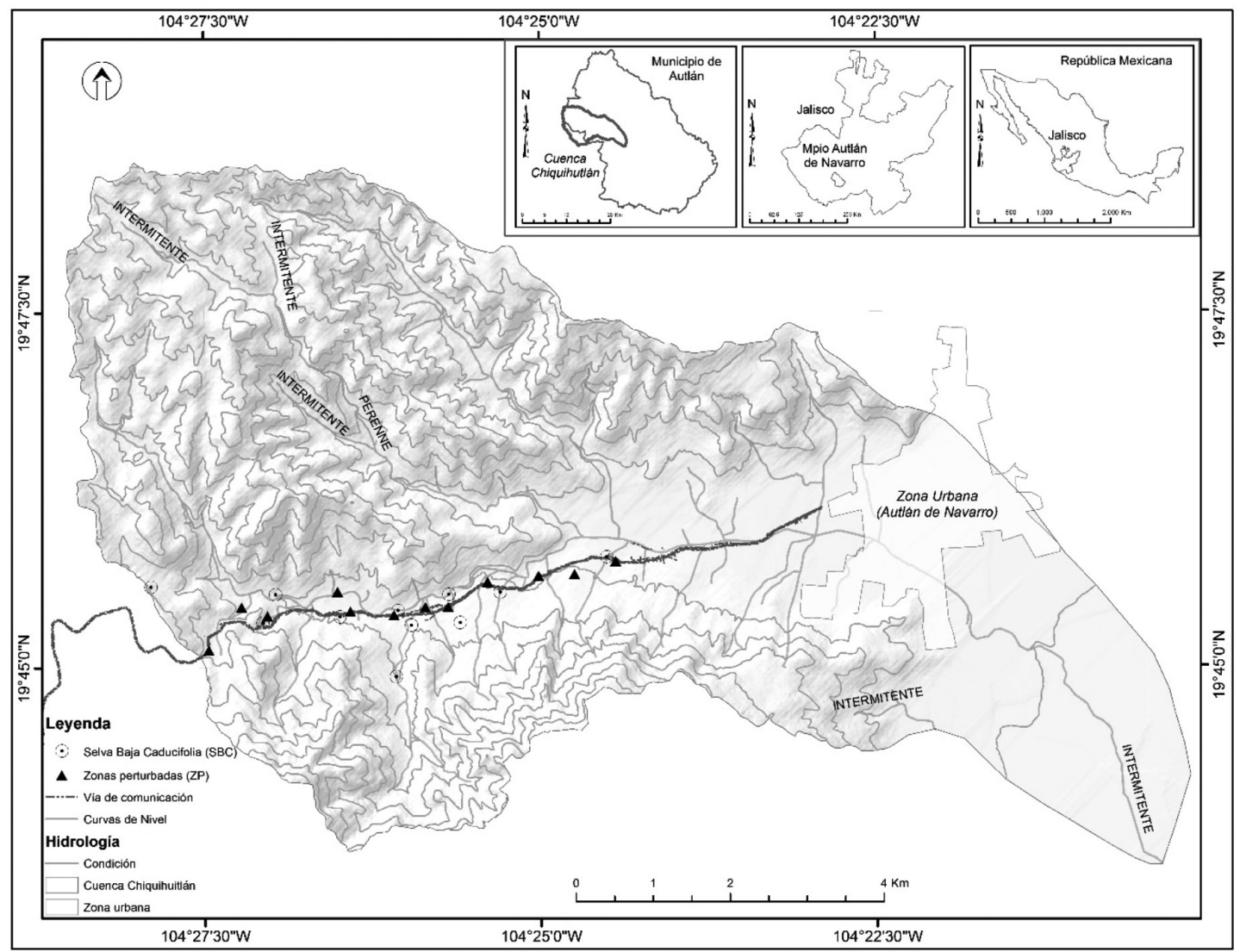

Figura 1. Ubicación de la zona de estudio.

las UM, fue estimada la riqueza específica del sitio mediante el índice de Margalef y la diversidad mediante Shannon-Wiener. El primero de ellos, riqueza específica de especies (Da) considera que valores $\leq 1,5$ reflejan baja riqueza, de 2,5 en adelante son considerados sitios de mayor riqueza (Margalef, 1977).

$$
\mathrm{Da}=\frac{\mathrm{S}-1}{\log \mathrm{N}}
$$

$\mathrm{Da}=$ Riqueza específica de especies; $\mathrm{S}=$ número de especies; $\mathrm{N}=$ número total de individuos.

La diversidad de Shannon-Wiener (H') mide el grado promedio de incertidumbre para predecir la especie a la que pertenece un individuo tomado al azar dentro de las UM. Siendo entonces que a mayor valor de H' mayor diversidad de especies.

$$
H^{\prime}=\sum_{i=1}^{\mathrm{s}}(\rho \mathrm{i})\left(\log _{2} \rho \mathrm{i}\right)
$$

$\mathrm{H}=$ número de especies; $\mathrm{pi}=$ Proporción de individuos de las especies $i$ respecto al total de individuos (abundancia relativa de la especie $i$ ).

Todos los individuos colectados fueron preservados en frascos con alcohol al 70\%. Lombrices y enquitreidos fueron conservados en formol al $4 \%$. Para conocer las diferencias entre UM se aplicó una prueba $t$ de student mediante el paquete estadístico SPSS Versión 21.0.

\section{Resultados y discusión}

\section{Inventario de macroinvertebrados}

En las UM se colectaron un total de 325 individuos (Figura 2), la mayor densidad fue en la SBC con 230 macroinvertebrados (197 en hojarasca y 33 en suelo) distribuidos en 14 órdenes, predominando: Coleóptera (26\%), Hemiptera (20\%), Araneae (19\%) e Hymenoptera (7.3\%) Los meses de mayo y diciembre presentaron el mayor 


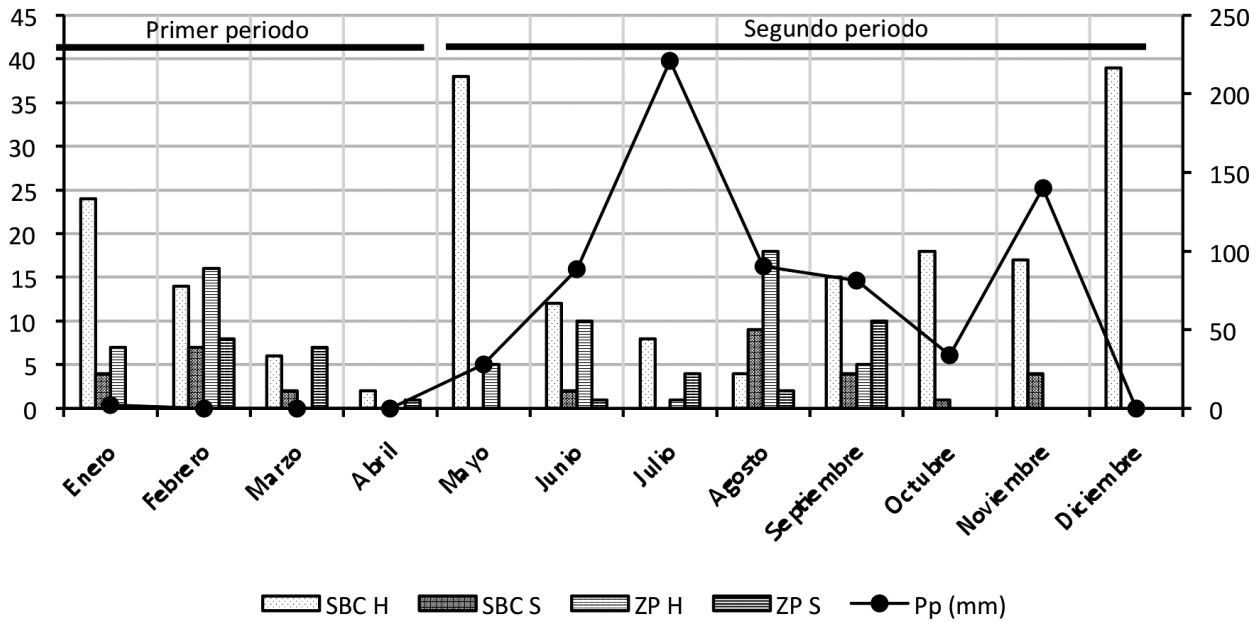

Figura 2. Densidad total y riqueza de individuos por UM en el año 2014.

número de individuos (38 y 39 respectivamente), los órdenes Araneae y Coleóptera registraron la mayor riqueza con 25 y 23 individuos respectivamente; febrero, junio, agosto y septiembre se registraron macroinvertebrados en ambos estratos de las UM.

En ZP se colectaron 95 organismos (62 hojarasca y 33 en suelo), representados por 14 órdenes dominando: Coleóptera (24\%), Hemiptera (20\%), Orthoptera (10\%), Araneae y Lepidoptera (7\% c/u) y Rhinodrilidae (6\%) (Tabla 1). Ge et al. (2012) reconocen que la menor intervención del ser humano o movimiento en ecosistemas o nula alteración del suelo favorece el establecimiento del mayor número de unidades taxonómicas, ya que provee protección, humedad constante y fuentes de alimentos en diferentes grados de descomposición para los macroinvertebrados.
La similitud en las UM fue de 6 órdenes y la disimilitud de 8 ( 3 para hojarasca y 5 en suelo), algunos de estos órdenes (Hymenoptera, Geophilomorpha, Haplotaxida, Mantodea) no son dominantes en riqueza, no obstante son representativos dentro de las UM; las diferencias proporcionales entre hojarasca y suelo, son en SBC de 6:1 y para ZP 2:1. La proporción encontrada entre SBC y ZP en suelo fue de 1:1 y en hojarasca 3:1, resaltando los taxones: Araneae, Coleóptera, Hemíptera, Hymenoptera, Orthoptera y Lepidoptera (este último en su totalidad en estadío larvario). Más del 50\% de la densidad de individuos en ambos estratos fue dominado por los taxones Coleóptera, Hemíptera y Araneae. De estos órdenes 8 presentan similitud entre hojarasca y suelo y 6 presentan disimilitud (5 para hojarasca y uno en suelo). Se

Tabla 1. Órdenes encontrados en las UM.

\begin{tabular}{llll}
\hline \multicolumn{1}{c}{$S B C h$} & \multicolumn{1}{c}{$S B C s$} & \multicolumn{1}{c}{$Z P h$} & \multicolumn{1}{c}{$Z P s$} \\
\hline Araneae & Araneae & Araneae & Araneae \\
Blattodea & Chilopoda & Coleóptera & Coleóptera \\
Coleóptera & Coleóptera & Gastropoda & Dermaptera \\
Dermaptera & Gastropoda & Hemiptera & Embioptera \\
Diptera & Lepidoptera & Mantodea & Haplotaxida \\
Gastropoda & Orthoptera & Polydesmida & Hymenoptera \\
Hemiptera & Geophilomorpha & Orthoptera & Diptera \\
Hymenoptera & Hemiptera & Lepidoptera & Lepidoptera \\
Lepidoptera & Haplotaxida & Hymenoptera & Geophilomorpha \\
Orthoptera & & & Hemíptera \\
Mantodea & & & \\
Opiliones & & & \\
Spirobolida & & & \\
\hline
\end{tabular}


identificaron 37 familias (27 para hojarasca y 10 en suelo) predominando en hojarasca: Araneidae $(21,8 \%)$, Staphylinidae (13,7\%), Reduvidae (3\%), Acrididae (2,5\%); en suelo: Scarabaeidae (33\%), Mecistocephalidae (15\%) y Rhinodrilidae (12\%). Se identifica que en el estrato de hojarasca $(h)$ algunos de los sitios de muestreo de ZP no hubo presencia de hojarasca lo que puede explicar las diferencias marcadas entre $\mathrm{SBCh}$ vs. $\mathrm{ZPh}$.

De acuerdo al estadístico $t$ se encuentra diferencias significativas en SBCh Vs. ZPh y ZPs $(\alpha=0.05)$, para SBCs con respecto a $Z P h$ y ZPs no se encontraron diferencias significativas.

Estos resultados quedan enmarcados por dos periodos en que se distribuyó la precipitación pluvial, el primero de ellos (enero a abril) caracterizado por la escasa precipitación pluvial y la reducción de individuos colectados, y el segundo por la tendencia positiva entre el número de individuos y la precipitación pluvial.

Se contabilizaron 30 familias en SBC 19 en hojarasca y 11 en suelo predominando en hojarasca: Acrididae (11\%), Lepidoptera (9\%), Reduvidae, Araneidae y Tenebrionidae (7\% c/u),
Carabidae, Gryllidae y Lygaeidae $(6 \% \mathrm{c} / \mathrm{u})$. Y en suelo: Tenebrionidae y Rhinodrilidae $(18 \% \mathrm{c} / \mathrm{u})$, Lygaeidae (9\%) y Araneidae y Mecistocephalidae $(6 \% \mathrm{c} / \mathrm{u})$ (Tabla 2).

\section{Riqueza y diversidad de UM}

El mayor registro de riqueza (Da) estimada fue para ZP con 3,26, valor considerado de alta riqueza lo que hace suponer que la alteración de esta UM por efecto de la construcción de vías de comunicación promueven establecimiento de especies y número de individuos, posiblemente por la remoción de material de suelo y vegetal, condición que promueve nuevas estructuras o hábitats. En consideración a los estratos de las UM, la respuesta de Da fue superior en hojarasca SBC con 3,59 respecto $Z P$ en suelo con 3,43; en esta última unidad se registró baja abundancia de individuos pero alta diversidad, condición que hace afirmar que esta unidad es más rica en comparación con la SBC (Tabla 3). Los valores mínimos observados en las UM a pesar de ser inferiores a los máximos registrados, se consideran elevados (Margalef,

Tabla 2. Familias encontradas en las UM.

\begin{tabular}{|c|c|c|c|}
\hline SBCh & SBCs & $Z P h$ & $Z P s$ \\
\hline $\begin{array}{l}\text { Acrididae } \\
\text { Alydidae } \\
\text { Anthocoridae } \\
\text { Araneidae } \\
\text { Beretidae } \\
\text { Carabidae } \\
\text { Chrysomelidae } \\
\text { Cicadelidae } \\
\text { Coosmetidae } \\
\text { Curculionidae } \\
\text { Dryopipidae } \\
\text { Forficulidae } \\
\text { Gryllidae } \\
\text { Mantidae } \\
\text { Mecistocephalidae } \\
\text { Mellolontidae } \\
\text { Mutillidae } \\
\text { Otitidae } \\
\text { Pentatomidae } \\
\text { Phalangidae } \\
\text { Lygaeidae } \\
\text { Reduvidae } \\
\text { Simulidae } \\
\text { Spirobolidae } \\
\text { Staphylinidae } \\
\text { Stygnommatidae } \\
\text { Tenebrionidae }\end{array}$ & $\begin{array}{l}\text { Araneidae } \\
\text { Buprestidae } \\
\text { Carabidae } \\
\text { Formicidae } \\
\text { Gryllidae } \\
\text { Lumbricidae } \\
\text { Mecistocephalidae } \\
\text { Naucoridae } \\
\text { Scarabaeidae } \\
\text { Tenebrionidae }\end{array}$ & $\begin{array}{l}\text { Acrididae } \\
\text { Araneidae } \\
\text { Carabidae } \\
\text { Chrysomelidae } \\
\text { Eumolpinae } \\
\text { Formicidae } \\
\text { Gryllidae } \\
\text { Litugusidae } \\
\text { Lygaeidae } \\
\text { Mantidae } \\
\text { Mecistocephalidae } \\
\text { Reduvidae } \\
\text { Scuterellidae } \\
\text { Scarabaeidae } \\
\text { Tenebrionidae } \\
\text { Xystodesmidae }\end{array}$ & $\begin{array}{l}\text { Araneidae } \\
\text { Eumolpinae } \\
\text { Forficulidae } \\
\text { Formicidae } \\
\text { Lumbricidae } \\
\text { Lygaeidae } \\
\text { Mecistocephalidae } \\
\text { Naucoridae } \\
\text { Sacarabaeidae } \\
\text { Tenebrionidae }\end{array}$ \\
\hline
\end{tabular}


Tabla 3. Valores de riqueza y diversidad por UM.

\begin{tabular}{lcccc}
\hline $\begin{array}{c}\mathrm{N}^{\mathrm{o}} \\
\text { individuos/Índices }\end{array}$ & $\begin{array}{c}S B C \\
(h)\end{array}$ & $\begin{array}{c}S B C \\
(s)\end{array}$ & $\begin{array}{c}Z P \\
(h)\end{array}$ & $\begin{array}{c}Z P \\
(s)\end{array}$ \\
\hline $\mathrm{UM}$ & 197 & 33 & 62 & 33 \\
Órdenes & 14 & 8 & 9 & 10 \\
Da General & 3,17 & & 3,26 & \\
$\mathrm{Da}$ & 3,59 & 2,50 & 2,67 & 3,43 \\
$\mathrm{H}$ & 2,98 & 2,44 & 2,60 & 2,80 \\
\hline
\end{tabular}

1977). Ge et al. (2012) reportaron valores de Da superiores a 10 en zonas perturbadas urbanas.

La diversidad biológica $\left(H^{\prime}\right)$ de las UM fue superior en SBCh $(2,98)$ y ZPs $(2,80)$, no obstante los valores mínimos registrados para SBCs y ZPh son considerados ricos ante la composición de cada una de ellas. Respuesta inferior al reporte de $\mathrm{Ge}$ et al. (2012) en zonas perturbadas y no perturbadas, registrando valores de $\mathrm{H}^{\prime}$ de 4,5 , resultados que manifiestan que las condiciones del sitio (espacial y temporal) y la estructura de cobertura, pueden ser condicionantes para el establecimiento de macroinvertebrados y ante ello de su diversidad biológica y abundancia estacional.

\section{Grupos funcionales}

La composición funcional de los macroinvertebrados en cada UM, es manifiesta a través de la presencia o ausencia de individuos en consideración del orden y familia, tomando en cuenta funciones ecosistémicas más generales como: ingenieros del suelo, detritívoros, herbívoros y depredadores (Cabrera et al., 2011).

\section{Ingenieros del suelo}

De los registros obtenidos se identifica que la familia más dominante es Formícidae; Cabrera et al. (2011) encontraron que ésta tiene un impacto específico en el interior del suelo a partir de la transformación de sus propiedades físicas, que favorecen la formación de agregados y mejoramiento de la estructura, facilitando el intercambio gaseoso, el movimiento y retención del agua (Tabla 4).

La presencia o ausencia de la familia Rhinodrilidae (lombrices de tierra) en las UM, es influida por el nivel de intervención antrópica, la intensidad de uso de la tierra y el grado de perturbación del medio edáfico, lo que confirma
Tabla 4. Grupo funcional ingenieros del suelo

\begin{tabular}{clcccc}
\hline \multicolumn{1}{c}{ Orden } & \multicolumn{1}{c}{ Familia } & $S B C h$ & $S B C s$ & $Z P h$ & $Z P s$ \\
\hline Haplotaxida & Lumbricidae & & $\mathrm{X}$ & & $\mathrm{X}$ \\
\hline \multirow{2}{*}{ Hymenoptera } & Formícidae & $\mathrm{X}$ & $\mathrm{X}$ & $\mathrm{X}$ & $\mathrm{X}$ \\
\cline { 2 - 6 } & Mutillidae & $\mathrm{X}$ & & & \\
\hline Coleóptera & Melolonthidae & $\mathrm{X}$ & & & \\
\hline Diptera & Simulidae & $\mathrm{X}$ & & & $\mathrm{X}$ \\
\hline
\end{tabular}

Rodríguez (2000); adicional a ello, se identificó que el incremento de la inclinación del terreno ( $\geq 35 \%$ de pendiente), la reducción de la materia orgánica y humedad en el suelo ( $<100$ centibares) disminuye la presencia de individuos de esta familia. Se afirma que en zonas perturbadas las lombrices del suelo como ingenieros de suelo son considerados los más importantes por encontrarse en ambos estratos de las UM, ello a pesar de la compactación del suelo, afectando su riqueza y abundancia en tiempo y espacio.

\section{Detritívoros}

Los Tenebrionidae fue la familia más representativa de este grupo, así como la clase Mollusca (Tabla 5); otras familias encontradas fueron: Planorbidae, Staphylinidae, Tenebrionidae, Forficulidae, Xystodesmidae, Atopetholidae, Spirobolidae. Rodríguez et al. (2002) identifica que este grupo puede ser afectado debido a los diferentes grados de perturbación de los estratos suelo y vegetación, siendo la relación $\mathrm{C} / \mathrm{N}$ factor

Tabla 5. Grupo funcional Detritívoros.

\begin{tabular}{llcccc}
\hline Orden y clase & Familia & $S B C h$ & $S B C$ s & $Z P h$ & $Z P S$ \\
\hline Mollusca & Planorbidae & $\mathrm{X}$ & $\mathrm{X}$ & $\mathrm{X}$ & \\
\hline Blattodea & & $\mathrm{X}$ & & & \\
\hline Coleóptera & Sthaphylinidae & $\mathrm{X}$ & & & \\
\cline { 2 - 6 } & Tenebrionidae & $\mathrm{X}$ & $\mathrm{X}$ & $\mathrm{X}$ & $\mathrm{X}$ \\
\hline Dermaptera & Forficulidae & $\mathrm{X}$ & & & $\mathrm{X}$ \\
\hline Polydesmida & Xystodesmidae & & & $\mathrm{X}$ & \\
\hline Spirobolida & Atopetholidae & $\mathrm{X}$ & & & \\
\cline { 2 - 6 } & Spirobolidae & $\mathrm{X}$ & & & \\
\hline
\end{tabular}


definitivo para su establecimiento. Zerbino et al. (2008) enfatizan a los detritívoros como organismos desprotegidos en la superficie del suelo, que se reducen drásticamente por las variaciones bruscas en las condiciones de temperatura y humedad debido a la menor cobertura y cantidad de residuos, y a una mayor exposición a la radiación solar en aquellos ecosistemas alterados.

\section{Herbivoros}

Este grupo fue de los más representativos en abundancia y riqueza en tiempo y espacio, identificando 6 órdenes y 16 familias (Tabla 6), los órdenes más distintivos fueron: Hemíptera con 6 familias y Coleóptera 5 familias. Las familias más dominantes en todos los estratos fueron: Scarabaeidae y Chrysomelidae (orden Coleóptera), Gryllidae (orden Orthoptera), Lygaeidae (orden Hemiptera) y otras no identificadas del orden Lepidoptera. Se resaltan las familias Eumolpinae, Forficulidae, Scuterellidae y Tetrigidae como exclusivas de ZP, lo que confirma esta UM promotora de diversidad biológica en ecosistemas antropizados.

Tabla 6. Grupo funcional Herbívoros.

\begin{tabular}{|c|c|c|c|c|c|}
\hline Orden & $\begin{array}{c}\text { Familia y } \\
\text { subfamilia }\end{array}$ & $S B C h$ & $S B C s$ & $Z P h$ & $Z P s$ \\
\hline \multirow{5}{*}{ Coleóptera } & Chrysomelidae & $\mathrm{x}$ & $\mathrm{x}$ & $\mathrm{x}$ & \\
\hline & Buprestidae & $\mathrm{x}$ & & & \\
\hline & Curculionidae & $\mathrm{x}$ & & & \\
\hline & Scarabaeidae & $\mathrm{x}$ & $\mathrm{x}$ & $\mathrm{x}$ & $\mathrm{X}$ \\
\hline & Eumolpinae & & & $\mathrm{x}$ & $\mathrm{X}$ \\
\hline Dermaptera & Forficulidae & & & & $X$ \\
\hline Diptera & Otitidae & $\mathrm{x}$ & & & $\mathrm{X}$ \\
\hline \multirow{6}{*}{ Hemiptera } & Pentatomidae & $\mathrm{x}$ & & & \\
\hline & Cicadelidae & $\mathrm{x}$ & & & \\
\hline & Lygaeidae & $\mathrm{x}$ & & $\mathrm{x}$ & $\mathrm{X}$ \\
\hline & Berytidae & $\mathrm{x}$ & & & \\
\hline & Alydidae & $\mathrm{x}$ & & $\mathrm{x}$ & \\
\hline & Scuterellidae & & & $\mathrm{x}$ & \\
\hline Lepidóptera & & $\mathrm{x}$ & $\mathrm{x}$ & $\mathrm{x}$ & $X$ \\
\hline \multirow{3}{*}{ Orthoptera } & Acrididae & $\mathrm{x}$ & & $\mathrm{x}$ & \\
\hline & Gryllidae & $\mathrm{x}$ & $\mathrm{x}$ & $\mathrm{x}$ & \\
\hline & Tetrigidae & & & & $\mathrm{X}$ \\
\hline
\end{tabular}

\section{Depredadores}

Este grupo de gran importancia ecológica y económica, estuvo representado por 7 órdenes y 12 familias, siendo los más representativos en abundancia y riqueza: Opiliones (exclusivos de SBCh) y Hemíptera con 3 familias cada uno. Los de mayor dominancia en los estratos fueron: Araneae (taxón fundamental por controlar la sobrepoblación de insectos y artrópodos plaga y localizados en todos los meses de muestreo en $\mathrm{SBCh}$ ) y la familia Mecistocephalidae del orden Geophilomorpha. Taxones exclusivos de ZPh se encontraron las familias: Scolopendridae del orden Scolopendromorpha, Henicopidae del orden Lithobiomorpha, Reduviidae del orden Hemiptera y Liturgusidae del orden Mantodea (Tabla 7). Zerbino et al. (2008) reconocieron que los depredadores presentan una mayor riqueza y abundancia en los sistemas de cultivos continuos. Resultados que difieren de Lee (1985) que identificó que en los sistemas de cultivos anuales intensivos se reducen las poblaciones de depredadores. Respuesta que puede estar relacionada al manejo convencional de productos agroquímicos.

\section{Conclusiones}

Se reconoce que las condiciones físicas del suelo como el grado de compactación efecto del tránsito de maquinaria pesada, reducción de la cobertura vegetal y humedad en el suelo, así como el cambio de uso de suelo a sistemas agrícolas (quemas agrícolas y establecimiento de coamiles) y pecuarios (siembra de pastos y presencia de ganado) son condiciones que propician el intemperismos y/o degradación del material edáfico.

La Da de las UM no muestran diferencias estadísticas marcadas, considerándose estas como sitios de alta riqueza y diversidad, respuesta que se incrementa con la presencia del temporal de lluvias y/o la presencia de humedad en el ambiente y suelo (junio a diciembre).

La variedad de órdenes y familias encontradas en los grupos funcionales fue diversa, identificándose plagas agrícolas de suelo y cultivo, así como depredadores de éstos o controladores biológicos e indicadores de calidad ambiental; sobresaliendo por su importancia en el sistema suelo los identificados como ingenieros del mismo. 
Tabla 7. Grupo funcional Depredadores.

\begin{tabular}{|c|c|c|c|c|c|}
\hline Orden & $\begin{array}{l}\text { Familia y } \\
\text { subfamilia }\end{array}$ & $S B C h$ & SBCs & $Z P h$ & $Z P s$ \\
\hline Araneae & & $\mathrm{x}$ & $\mathrm{x}$ & $\mathrm{x}$ & $X$ \\
\hline \multirow{3}{*}{ Opiliones } & Stygnommatidae & $\mathrm{x}$ & & & \\
\hline & Phalangiidae & $\mathrm{x}$ & & & \\
\hline & Cosmetidae & $\mathrm{x}$ & & & \\
\hline Coleóptera & Carabidae & $\mathrm{x}$ & $\mathrm{x}$ & $\mathrm{x}$ & \\
\hline Geophilomorpha & Mecistocephalidae & $\mathrm{x}$ & $\mathrm{x}$ & $\mathrm{x}$ & $\mathrm{X}$ \\
\hline Scolopendromorpha & Scolopendridae & & & $\mathrm{x}$ & \\
\hline Lithobiomorpha & Henicopidae & & & $\mathrm{x}$ & \\
\hline \multirow{3}{*}{ Hemiptera } & Anthocoridae & $\mathrm{x}$ & & $\mathrm{x}$ & \\
\hline & Naucoridae & & $\mathrm{x}$ & & \\
\hline & Reduviidae & & & $\mathrm{x}$ & \\
\hline \multirow{2}{*}{ Mantodea } & Mantidae & $\mathrm{x}$ & & $\mathrm{x}$ & \\
\hline & Liturgusidae & & & $x$ & \\
\hline
\end{tabular}

La construcción de vías de comunicación contrario a lo que se pudiera pensar, no afectó la riqueza y diversidad de las UM, pero si en la composición de estas en comparación con la SBC, favoreciendo el establecimiento de individuos generalistas en ZP. Los suelos de estos sitios fueron caracterizados por su compactación, degradación y reducida integración de material orgánico (hojarasca). El potencial de adaptación de estos organismos a cambios drásticos en su hábitat natural representa la creación de nuevos nichos para la recolonización de nuevos hábitats o la adaptación de otros organismos a dichos cambios, impactando la similitud entre UM.

El mayor registro de individuos fue para los grupos funcionales herbívoros y depredadores; considerados como los grupos generalistas con mayor grado de adaptación a establecerse en ZP con tendencias de cambio de uso de suelo (agropecuario) y deforestación; los grupos con menores registros fueron los ingenieros del suelo y detritívoros.

Se considera importante promover investigaciones referidas a las funciones ecosistémicas e importancia de estos organismos en diferentes hábitats y su relación a la perturbación de estas; así como su relación con parámetros físico - químicos del suelo y ambientales. Cabe mencionar que el estudio de la biología del suelo enfocado a los macroinvertebrados ha sido poco explorado en México lo cual demanda promover y realizar más estudios acerca del tema mediante el establecimiento de sitios permanentes.

\section{Literatura Citada}

Begon, M., Harper, J.L. y Townsend, C.R.

1999. Ecología: individuos, poblaciones y comunidades Ediciones Omega. Barcelona, España, 1172 p.

Brown, G.G.C. Fragoso, I., Baroi, P., Roja, C.J., Patrón. J.L., Bueno, G.A., Moreno, P., Lavelle, V. y Orda, R.C.

2001. Diversidad y rol funcional de la macrofauna edáfica en los ecosistemas tropicales mexicanos. Acta Zoológica Mexicana, (n.s.). Número especial, 1: 79-110.

Brussard, P.F., Reed, J.M., y Tracy, C.R.

1998. Ecosystem management: what is it really?. Landscape and Urban Planning, 40(1): 9-20.
Cabrera, G., Robaina, N., Ponde de León, D.

2011 Composición funcional de la macrofauna edáfica en cuatro usos de la tierra en las provincias de Artemisa y Mayabeque. Cuba. Pastos y Forrajes., 34(3): 331-346.

Ge, B.M., Li, Z.X., Zhang, D.Z., Zhang, H., Liu, Z.T., Zhou, C.L.; Tang, B.

2012. RESEARCH ARTICLE Communities of soil macrofauna in green spaces of an urbanizing city at east China. Revista Chilena de Historia Natural, 85(2): 219-226. 
Guinchard, M.; Robert, J.C.

1991. Approche biocénotique du système sol par l'étude du peuplement de larvesd'insectes (première contribution). Revue D'Ecologie et de Biologie du Sol, 28: 479-490.

Hooper, D.U., Solan, M., Symstad, A., Diaz, S., Gessner, M.O., Buchmann, N.; Roy, J.

2002. Species diversity, functional diversity and ecosystem functioning. Biodiversity and Ecosystem Functioning: Syntheses and Perspectives, 17: 195-208.

Jones, C.G., Lawton, J.H.; Shachak, M.

1994. Organisms as ecosystem engineers. In: Samson, F. B. y Knopf, F.L. Ecosystem management. Springer. New York, US. pp. 130-147.

Jones, C.G., Lawton, J.H.; Shachak, M.

1997. Positive and negative effects of organisms as physical ecosystem engineers. Ecology, 78(7): 1946-1957.

Lavelle, P., Decaëns, T., Aubert, M., Barot, S., Blouin, M.,

Bureau, F.; Rossi, J.P.

2006. Soil invertebrates and ecosystem services. European Journal of Soil Biology, 42: S3-S15.

Lavelle, P., Lepage, M.; Cambefort, Y.

1993. Soil Macrofauna. IN: Ingram, J.S.i. y Anderson, J.M.(eds). Tropical Soil Biology and Fertility. A Handbook of Methods. CAB international. Wallingford, UK. pp. 44-46.

Lavorel, S.; Garnier, E.

2002. Predicting changes in community composition and ecosystem functioning from plant traits: revisiting the Holy Grail. Functional ecology, 16 (5): 545-556.

Lee, K.E.

1985. Earthworms: their ecology and relationships with soil and land use. Academic Press. New York, USA, 411 p.

Margalef, R. 1977. Ecología. Ediciones Omega. Barcelona, España, 951 p.

Rodríguez, C.

2000. Comunidades de lombrices de tierra en ecosistemas con diferente grado de perturbación. Rev. Biología. 14 (2): 147.

Rodríguez, I.; Crespo, G.; Rodríguez, C.; Castillo, E.; Fraga, S. 2002. Comportamiento de la macrofauna del suelo en pastizales con gramíneas naturales puras o intercaladas con leucaena para la ceba de toros. Revista Cubana de Ciencia Agrícola, 36(2): 181-186.
Rzedowski, J.

1991. El endemismo en la flora fanerogámica mexicana: una apreciación analítica preliminar. Acta Botánica Mexicana. 15: 47-64

Salamanca, N.; Chamorro, C.

1994. La edafofauna del páramo de Monserrate Sector Hacienda "Santa Bárbara", Cundinamarca Colombia. En: Mora Osejo, L.E. y Sturm, H. (Eds.). Estudios Ecológicos del Páramo y del Bosque Andino Cordillera Oriental de Colombia. Academia Colombiana de Ciencias Naturales Exactas, Físicas y Naturales. Santa Fe de Bogotá. Colombia. pp. 619-630.

Shannon, C.E.; Weaver, W.

1949. The mathematical theory of communication. University of Illinois Press. Urbana, IL, US, 144 p.

Shugart, H.H.; Woodward, F.I.

1997. Plant functional types: their relevance to ecosystem properties and global change (Vol. 1). Cambridge University Press. Cambridge, UK, 369 p.

Stork, N.E. Eggleton, P.

1992. Invertebrates as Determinants and Indicators of Soil Quality. American. Journal of Alternative Agriculture, 7(1-2): 38-45.

Trejo, I.; Dirzo, R.

2000. Deforestation of seasonally dry tropical forest: a national and local analysis in Mexico.Biological Conservation, 94(2): 133-142.

Wolters, V.; Ekschmitt, K.

1997. Gastropods, Isopods, Diplopods, and Chilopods: Neglected Groups of the Decomposer Food Web. In: Benckiser, G. (Ed.). Fauna in Soil Ecosystems: Recycling Processes, Nutrient Fluxes, and Agricultural Production. Marcel Dekker. Inc. New York, US. 265-306 pp.

Zaldívar, N., Pérez, B. E., Fernández, Y.; Licea, L. 2007. Macrofauna edáfica en tres sistemas ganaderos. Centro Agrícola, 34(2): 75-79.

Zerbino, S., Altier, N., Morón, A.; Rodríguez, C.

2008. Evaluación de la macrofauna del suelo en sistemas de producción en siembra directa y con pastoreo. Agrociencia, 12(1): 44-55. 
